\title{
Linx
}

Revue des linguistes de l'université Paris X Nanterre

$8 \mid 1996$

Du dire et du discours

\section{Interdiscours et construction de l'objet de discours}

\section{Frédérique Sitri}

\section{OpenEdition}

\section{Journals}

Édition électronique

URL : http://journals.openedition.org/linx/1158

DOI : 10.4000/linx.1158

ISSN : 2118-9692

\section{Éditeur}

Presses universitaires de Paris Nanterre

\section{Édition imprimée}

Date de publication : 1 septembre 1996

Pagination : 153-172

ISSN : 0246-8743

\section{Référence électronique}

Frédérique Sitri, «Interdiscours et construction de l'objet de discours », Linx [En ligne], $8 \mid$ 1996, mis en ligne le 24 juillet 2012, consulté le 23 avril 2019. URL : http://journals.openedition.org/linx/1158; DOI : $10.4000 / \operatorname{linx} .1158$

Ce document a été généré automatiquement le 23 avril 2019.

Département de Sciences du langage, Université Paris Ouest 


\title{
Interdiscours et construction de l'objet de discours
}

\author{
Frédérique Sitri
}

1 L'analyse de discours apparaît aujourd'hui comme un champ «éclaté »1, en relation de filiation problématique avec une « Ecole française d'analyse du discours » souvent décrite comme monolithique ${ }^{2}$. Nous poserons ici la question de l'articulation de notions issues de I'“AD française" avec une recherche située dans un cadre théorique quelque peu différent : l'étude de l'objet de discours dans un corpus oral de situations argumentatives. Après avoir rapidement présenté les notions sur lesquelles nous nous appuyons, nous soumettrons à la réflexion du lecteur quelques exemples précis d'analyses tirés du corpus sur lequel nous travaillons.

\section{Préliminaires théoriques et méthodologiques}

\section{L'objet d'étude}

2 Alors que l'AD française privilégiait, en conformité avec son projet politique, les corpus écrits ${ }^{3}$ à dimension idéologique forte, depuis quelque temps se multiplient les analyses de corpus oraux ${ }^{4}$ qui peuvent être de nature fort diverse : monologues ou dialogues (voire « trilogues »), produits dans un cadre professionnel ou privé, avec ou sans répartition des tours de parole ...

3 Le corpus d'étude a été construit à l'origine pour étudier l'argumentation orale en interaction. Au sein de réunions à visée de régulation ou de négociation, se tenant dans un cadre professionnel ou associatif, sont découpées des séquences dont l'unité est fournie par une "question argumentative $»^{5}$, autour de laquelle vont s'affronter argumentaire et contre-argumentaire. C'est à travers l'étude des phénomènes de construction et de transformation de l'objet de discours qu'est abordé ce matériel. 


\section{La notion d'objet de discours}

4 Un des soubassements théoriques du travail est constitué par la notion d'objet de discours, telle qu'elle est formalisée par la logique naturelle ${ }^{6}$. Dans ce cadre, l'objet de discours est défini comme un "faisceau d'objet " ${ }^{7}$, c'est-à-dire "un ensemble d'aspects normalement attachés à l'objet $»^{8}$. Ce faisceau, qui se construit dans le discours, se déploie sous la forme d'une "classe-objet »". Différentes opérations logico-discursives, qui sont la trace d'une activité de schématisation, permettent d'ancrer un objet dans le discours à partir d'une notion primitive, puis de passer d'un ingrédient à l'autre de la classe-objet.

$5 \quad$ L'étude de la structuration de l'objet de discours dans le corpus décrit plus haut s'élabore autour de deux questions-clés.

6 1. La question des marques permettant de repérer un objet de discours ${ }^{10}$. Nos analyses nous amènent à prendre en considération un faisceau de trois types de marques :

- les marques de thématisation

- les marques de problématisation ${ }^{11}$

- les marques de ratification ${ }^{12}$

7 2. La question des effets discursifs liés à la construction de l'objet. Les effets liés à la présence de tel ou tel « désignateur » ont été étudiés dans différents types de discours. Dans une perspective argumentative, l'enjeu en est, nous semble-t-il, d'autant plus crucial. C'est dans la description de ce type de phénomènes qu'est apparu le rapprochement possible avec des notions issues de l'AD - préconstruit, interdiscours, mémoire discursive et domaine de mémoire.

\section{Préconstruit, interdiscours et mémoire discursive}

$C^{\prime}$ est la notion $\mathrm{d}^{\prime}$ « interdiscours » qui est première en $\mathrm{AD}^{13}$. Mise en place assez tôt dans la théorie de M. Pêcheux, à partir de la réflexion sur le préconstruit, elle devient centrale dans ce que M. Pêcheux lui-même a appelé "la troisième époque» de l'Analyse du discours, rejoignant alors la notion d'« hétérogénéité constitutive » de J. Authier, et celle de « domaine de mémoire » chez J.J. Courtine.

9 Ainsi que le souligne D. Maldidier (1990), le préconstruit fournit « l'ancrage linguistique de la saisie de l'interdiscours » (26) défini, selon une formule maintes fois reprise, par le fait que "ça parle, avant, ailleurs et indépendamment» (43). C'est un travail sur les relatives ${ }^{14}$ qui amène $M$. Pêcheux à reformuler dans un cadre discursif la notion purement logique de "présupposé » élaborée par Ducrot :

Toute la réflexion menée avec $P$. Henry sur le préconstruit montrait les traces dans

le discours d'éléments discursifs extérieurs dont on a oublié l'énonciateur

(Maldidier, 1990, 43).

10 Les notions, liées, de " mémoire discursive » et de « domaine de mémoire » apparaissent quant à elles dans le travail de Courtine sur le discours communiste adressé aux chrétiens ${ }^{15}$, à partir d'une relecture de L'Archéologie du savoir ${ }^{16}$. Ainsi y-a-t-il surgissement d'une "mémoire discursive» quand, au milieu de l'événement discursif" que constitue le discours de G. Marchais aux Chrétiens le 10 juin 1976, apparaît, par l'intrusion de quelques intégristes, l'énoncé de Pie XI condamnant le communisme, proféré une quarantaine d'années auparavant. L'effet de cette mémoire discursive peut être saisi si l'on constitue un domaine de mémoire, ensemble de séquences discursives qui 
préexistent à la séquence de référence, visant à analyser les «effets de rappel, redéfinition, transformation, mais aussi les effets d'oubli, de rupture, de dénégation du déjà-dit » (Courtine, 1981, 56). C'est donc une notion qui permet d'« appréhender les fonctionnements discursifs d'enchâssement du préconstruit et d'articulation d'énoncés " (ibid), mais qui opère également un «bougé » de la notion d'interdiscours ${ }^{17}$, reformulé en « corps de traces formant mémoire » (Pêcheux in Maldidier, 1990, 290).

\section{Quelques exemples d'analyse}

11 Les exemples sélectionnés présentent des configurations diverses d'inscription de l'objet dans le discours. La désignation de l'objet de discours peut ainsi intervenir à différents moments de la séquence :

- au début, dans la première intervention;

- dans le déroulement de la séquence - et l'on s'intéresse alors à la série des éléments appartenant à la même classe-objet ;

- à la fin de la séquence : l'objet de discours, présenté sous la forme d'énoncés « descriptifs », est catégorisé in fine par un énoncé du type c'est un $X$.

12 La mise en évidence de l'effet discursif lié à la présence de tel ou tel désignateur nous amènera à prendre en compte le choix du lexique (exemples 3 et 4), mais également une nominalisation (exemple 2) et la présence d'une relative (exemple 1).

\section{«les questions de dernière minute » : relative et interdiscours ${ }^{18}$}

13 La séquence commence par une déclaration liminaire du proviseur (P) qui, ouvrant le conseil d'administration, annonce son refus de répondre aux questions posées par les parents (fcpe) et par la documentaliste (cdi) ${ }^{19}$ :

nous av= j'ai / reçu // quatre questions de la fcpe et une question // sur le cdi /// alors / puisque je on commence e à travailler avec le nouveau conseil d'administration / je voudrais quand même e donner un certain nombre de: de remarques et de dispositions /// je ne crois pas personnellement / à la valeur des questions de dernière minute qui ont pas pu être étudiées préalablement (...)

Ces « questions » constituent bien un objet de discours : il y a problématisation (proviseur et parents ne sont pas d'accord sur le fait de savoir si le premier doit ou non répondre à ce type de questions), et l'objet est repris, « travaillé » par les participants ${ }^{20}$.

\section{" questions diverses » ou « questions de dernière minute » ?}

Mais qu'en est-il du désignateur proposé par le locuteur ? Les premiers énoncés, donnés ci-dessus, ouvrent une classe-objet : \{quatre questions de la fcpe, une question sur le cdi, les questions de dernière minute qui ont pas pu être étudiées préalablement, ...\}. Sans aller plus loin, on accordera que le dernier élément cité constitue le désignateur " par excellence $»^{21}$ de l'objet construit par le discours de P. Il est en effet constitué en terme générique par des marques de généralisation que l'on repère au niveau des déterminants, de l'aspect verbal, ou des caractérisations ${ }^{22}$.

Pour autant, si l'on se représente de quoi il est question ici, à savoir les questions, non répertoriées à l'ordre du jour, posées à la fin d'une réunion de ce type, le terme qui vient tout naturellement à l'esprit est celui de questions diverses ${ }^{23}$. Il se trouve d'ailleurs que ce 
syntagme est en quelque sorte « disponible » pour ce locuteur, puisqu'il apparait dans la suite de l'intervention - appliqué par analogie à une autre situation :

alors je sais bien que que le mercredi à la chambre des députés il y a bien le les questions diverses / mais e pf vous connaissez tout l'intérêt des questions diverses à la chambre des députés

\section{Le rôle de la relative}

Partant de ce décalage entre le "désignateur» employé par le locuteur (questions de dernière minute) et ce qui nous semble le «terme propre » (questions diverses), on se posera la question des effets discursifs liés au premier. Nous prendrons comme point de départ l'effet le plus évident, à savoir la valeur appréciative négative du syntagme ${ }^{24}$. En ellemême, la caractérisation de dernière minute ne comporte pas une telle valeur ${ }^{25}$, qui naît donc en discours. La modalisation négative qui affecte le prédicat joue bien entendu un rôle : je ne crois pas personnellement à ... ne laisse pas attendre une suite positive. Mais il faut également prendre en compte la relative (qui n'ont pas pu être étudiées préalablement) et cela va nous amener à caractériser un deuxième type d'effet.

Du point de vue intonatif, la relative présente les traits suivants :

- on perçoit une pause entre le SN et la relative ;

- la relative n'est pas en intonation d'incise ;

- la relative semble modulée ;

- la hauteur du relatif est difficile à déceler, mais il ne semble pas en intonation haute ${ }^{26}$. Mise à part l'hésitation sur le dernier point, on aurait donc affaire à ce que M.A. Morel et A. Rialland appellent une $"$ relative appositive autonome $»^{27}$.

Examinons maintenant la relation de sens entre la relative et l'antécédent : on voit que la première reformule la caractérisation de dernière minute. Les questions sont de dernière minute, autrement dit elles n'ont pas pu être étudiées préalablement, sans que l'on puisse déterminer si la prédication contenue dans la relative justifie la caractérisation («parce que ») ou en tire les conséquences ( donc »). En même temps - en prenant en compte l'intonation sans rupture du segment qui précède la relative - on peut considérer que celle-ci justifie l'ensemble de la prise de position négative qui précède : je ne crois pas personnellement à la valeur des questions de dernière minute, puisqu'elles n'ont pas pu être étudiées préalablement.

\section{Relative et articulation d'énoncés}

Mais si la relative concourt à créer l'effet d'appréciation négative, elle introduit en même temps - en creux - une information supplémentaire, un argument (sur lequel elle repose), que l'on peut restituer sous la forme suivante: «une question doit être étudiée préalablement ». Or, à ce point de notre description, ce sont certaines analyses proposées par M. Pêcheux, dans un texte certes ancien, qui font signe. Ainsi, on peut dire que l'énoncé dégagé ci-dessus comme soubassement de la relative est présenté comme un " savoir » qui "fait retour ", comme une «évidence » qui est simplement rappelée, sous la forme du "chacun sait que, il est clair que $»^{28}$. C'est ce que Pêcheux appelle "articulation d'énoncés» ou "processus de soutien», qui est une des formes de détermination du discours par l'interdiscours. Il est alors intéressant de noter 
l'actualisation, dans la suite de l'intervention, d'un paradigme en relation avec l'énoncé dont la relative opère, « en creux », le rappel :

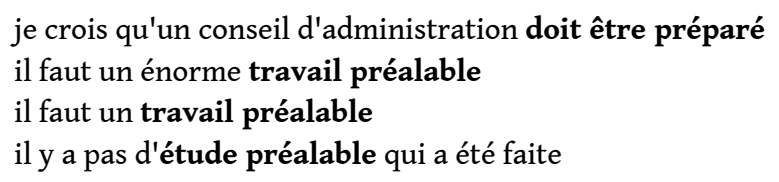

Nous ferons l'hypothèse, en dehors de tout moyen de saisie de l'interdiscours, que l'évidence ainsi imposée par la relative - et d'ailleurs non contestée par les participants est la trace d'un discours que nous appellerons «administratif» ou "institutionnel». Par-delà l'effet d'appréciation négative, dans la manière dont est posé l'objet de discours, on aurait donc un effet d'imposition d'une norme issue de l'interdiscours « administratif $»^{29}$.

\section{"la décision du conseil de classe » : nominalisation et interdiscours}

Dans ce deuxième exemple, l'effet d'imposition d'un préconstruit est lié à une nominalisation. P. Sériot, analysant le discours politique russe, a bien montré comment la nominalisation renvoie à « un objet du monde déjà-là, préexistant au discours ». Deux cas de figure peuvent être distingués : la nominalisation reformule un énoncé effectivement asserté, et « le préconstruit qu'elle manifeste est une anaphore » (Sériot, 1981, 30). Mais il y a aussi des "pseudo-anaphores, des préconstruits qui renvoient formellement à un discours antérieur, ce discours de référence pouvant aussi bien ne jamais avoir été tenu par le sujet de l'énonciation" (31). Ainsi la nominalisation contribue-t-elle "à faire d'un texte (...) une surface hétérogène où se mêlent et s'articulent des éléments de discours d'origine diverse $»(32)^{30}$.

\section{L'introduction de l'objet de discours}

Dans la séquence que nous étudions ${ }^{31}$, l'introduction de l'objet de discours est complexe :

G - - je reviens un petit peu en arrière sur l'article contrôle de la scolarité ce qui s'appelle vingt quatre mais qui serait vingt cinq plus tard (...) G - - bien alors la dernière phrase hein / (LIT) des bulletins trimestriels ou semestriels pour les sections de techniciens supérieurs + non attendez le conseil de classe / non c'est sur l'aspect conseil de classe alors les bulletins trimestriels qui coMportent la décision du coNseil de classe

On peut suivre le - laborieux - processus de formulation de l'objet à travers une série de thématisations, qui constituent une classe-objet. La première thématisation (sur l'article contrôle de la scolarité) permet le " raccrochage » à l'objet antérieur (l'article du règlement en discussion auparavant). On peut considérer la dernière phrase comme un "ingrédient " de cet objet, dont des bulletins trimestriels ... représente le déploiement en extension (il s'agit de la lecture de la phrase en question). Avec le conseil declasse, en revanche, est mis en avant un objet nouveau, quoique contigu au premier - d'où sans doute la focalisation c'est sur l'aspect conseil de classe. La dernière thématisation, les bulletins trimestiels qui comportent la décision du conseil de classe, opère alors selon nous un « saut qualitatif ». Si, en effet, sont repris deux éléments déjà posés, bulletin trimestriel et conseil de classe, ce dernier se trouve enchâssé dans le syntagme décision du conseil de classe. Ainsi un nouvel objet estil créé, au prix d'un "coup de force » qui tient aux effets discursifs propres à la nominalisation. 


\section{Effets de la nominalisation} décide (a décidé). Un tel énoncé n'apparaissant pas dans les échanges qui précèdent cette intervention, il ne s'agit donc pas d'une nominalisation de type anaphorique. Mais on peut aller plus loin et affirmer qu'une assertion telle que le conseil de classe décide a peu de chances d'apparaitre. En effet, les textes administratifs réglementant le fonctionnement des conseils de classe dénient à cette instance la capacité de décision - suscitant une vive contestation des enseignants, en désaccord avec cette réduction de leurs attributions et donc de leur pouvoir. En posant comme objet de discours le syntagme décision du conseil de classe, le locuteur (un professeur) impose donc comme préconstruit, comme déjà-là - a priori difficilement réfutable - un énoncé dont l'assertion est pour le moins problématique. L'acuité de la polémique explique peut-être que ce "coup de force» échoue : alors que dans le cas précédent l'imposition d'une norme de fonctionnement ne rencontrait pas de résistance, ici des réactions apparaissent immédiatement, sous la forme de deux énoncés :

il y a PAS de décisionc'est pas une décision

Si le premier énoncé réfute l'existence même de l'objet, le second refuse simplement la catégorisation opérée par le locuteur - autrement dit le préconstruit. On peut formuler l'hypothèse que le statut respectif des différents locuteurs n'est pas étranger à la réussite ou à l'échec de l'imposition d'un « objet de discours »32.

\section{Désignation et mémoire discursive}

Dans les deux cas qui suivent, c'est le lexème constituant le désignateur de l'objet que nous examinerons, en tant qu'il nous semble « convoquer » une « mémoire discursive ».

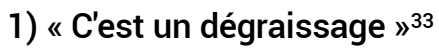

\section{La catégorisation de l'objet de discours}

Ici la construction de l'objet de discours est différente de ce qui a été vu dans les deux cas précédents : celui-ci est posé non pas sous la forme d'un désignateur mais d'un énoncé (donc une prédication), repris dans une série de reformulations :
a) depuis quelque temps à $\mathrm{X}$ des personnes sont contactées pour e savoir si ils désirent partir on leur fait des propositions on leur parle de fne $\mathrm{e}$
b) à l'heure actuelle il y a plusieurs dizaines de personnes qui sont contactées on leur fait des propositions / hein sur tant on vous propose tant est-ce que vous êtes prêt à partir et MEme des personnes qu'ont cinquante cinq ans
c) on contacte les gens on leur propose sans plan sans rien
d) et ça ces gens-là ils partent avec e certains avantages / on leur e fait un petit calcul et on leur demande s'ils veulent partir soit licenciement de gré à gré ou une démission / et ça c'est dans l'air du temps
e) non mais les personnes que je parlais tout à l'heure ils : voulaient pas partir / et on les a convoqués on leur a dit voilà on vous propose ça
f) mais la plupart du temps on appelle les gens et on leur dit

L'objet ainsi constitué pourrait être décrit comme un scénario en trois temps: /on contacte des personnes/,/on leur demande de partir/; /on leur fait des propositions 
financières $/{ }^{34}$. Puis ces reformulations font place, dans le déroulement de la séquence, à un énoncé d'allure différente ${ }^{35}$ :

en fait e c'est un dégraissage

qui opère une catégorisation ${ }^{36}$ de l'objet complexe présenté ci-dessus. Reprenant les analyses de Lecomte sur le discours du raisonnement, on verra dans l'apparition d'un tel énoncé un effet de dénivellement, de rupture (Lecomte parle aussi de " compactification»), lié à la forme c'est un $X$ ainsi qu'à la situation de l'énoncé dans la séquence ${ }^{37}$. Plus précisément, il s'opère comme un détachement, une mise à distance visà-vis d'un objet complexe dont on peut alors dire, sous la forme d'une évidence : «voilà ce que c'est, en fin de compte ».

\section{Effets de mémoire}

Le terme qui « catégorise » mérite donc qu'on s'y arrête : dégraissage renvoie au domaine de l'entreprise, où il est à peu près équivalent de licenciement ${ }^{38}$. On peut faire l'hypothèse que c'est un terme utilisé par les syndicats plus que par le patronat, et qu'il n'est pas seulement descriptif mais comporte une dimension de dénonciation : catégoriser ce qui se passe dans une entreprise comme étant un dégraissage, c'est rapprocher la situation particulière de cette entreprise d'autres situations analogues, connues et redoutées des salariés. Mais c'est aussi, dans le même temps, convoquer un ensemble de discours discours syndicaux certainement, mais aussi peut-être discours des médias - bref, une « mémoire discursive » dans laquelle le terme acquiert cette charge affective.

Il y a donc une dimension polémique dans la catégorisation effectuée par ce locuteur, qui peut expliquer l'effacement de l'agent opéré par la nominalisation ${ }^{39}:$ c'est en effet l'entreprise, le directeur assis en face qui procède à ce « dégraissage » de l'effectif. Il est intéressant alors de relever les énoncés qui constituent la réponse de ce dernier :

c'est bien un dégraissage si vous voulez

c'est une adaptation

c'est une adaptation à droite et à gauche des besoins

Après une première concession, le locuteur procède non pas à une réfutation pure et simple - comme dans le cas précédent - mais à une substitution qui propose une autre catégorisation du réel: dégraissage, terme évocateur d'une mémoire discursive, terme dénonciateur, est remplacé par adaptation, terme non chargé discursivement, "neutre ", simplement descriptif - technique voire technocratique.

\section{2) « le centralisme démocratique »}

33 L'objet auquel nous nous intéressons dans ce dernier exemple est quelque peu atypique. En effet, introduit à plusieurs reprises par un locuteur (A), non seulement il n'est pas repris mais il est même explicitement réfuté par la partie adverse (B), relayée par le médiateur ${ }^{40}$.

\section{Une classe-objet riche}

Du côté de $\mathrm{B}$, on observe que les termes renvoyant à l'objet la lettre sont pour l'essentiel en relation de reprise anaphorique : ce sont des désignateurs «neutres $»^{41}$. Chez $\mathrm{A}$, au contraire, on a une série beaucoup plus riche. Dans l'intervention initiale du locuteur, la classe-objet ${ }^{42}$ ainsi ouverte comprend les éléments suivants : \{cette lettre de septembre, ce 
procédé, le procédé utilisé, une lettre de dénonciation, cette lettre, le centralisme démocratique ${ }^{43}$. La première occurrence est une simple reprise anaphorique, caractérisée de manière neutre par une date (cette lettre de septembre). Mais l'objet est ensuite recatégorisé (comme le montre la présence de l'indéfini) par une lettre de dénonciation, en relation de coréférence avec le premier terme. Par ailleurs, un glissement s'opère de termes désignant l'objet lui-même dans sa matérialité (lettre) à des termes caractérisant le fait d'envoyer une lettre de ce type : le procédé, le centralisme démocratique.

Dans la suite de la séquence, la classe s'enrichit (outre de simples reprises anaphoriques) d'une série de termes renvoyant plutôt au contenu de la lettre (présence de dire) : \{un diktat, cette accusation grave, cette lettre, l'argument d'autorité, argument d'autorité et juridique / de type stalinien je dirais même\}.

\section{Effets de mémoire}

Là encore, on peut partir de l'effet le plus évident, la caractérisation négative de l'objet. Si chacun des termes pris en lui-même n'est pas nécessairement, en langue, porteur d'une valeur négative, discursivement, il est clair que l'ensemble concourt à produire cette impression.

La série \{centralisme démocratique, diktat, argument d'autorité\} en particulier apparaît liée par la notion d'autoritarisme. Mais un tel effet n'est possible que si l'on attribue au premier terme de la série, qui semble «engendrer» les autres, une telle valeur. Or le terme centralisme démocratique n'est pas nécessairement négatif. Dans un autre discours, il peut être purement descriptif, voire technique : il renvoie au mode de fonctionnement d'une organisation politique. Pour qu'il forme une "constellation" avec diktat et argument d'autorité, il est nécessaire que soit convoqué un type de discours qui attribue à centralisme démocratique cette valeur négative d'autoritarisme - disons pour faire vite le discours anti-communiste.

Cette interprétation est renforcée par l'apparition, un peu plus loin, de l'adjectif stalinien, dont la portée négative est pratiquement admise nous semble-t-il en langue ${ }^{44}$. On peut reconsidérer également la catégorisation opérée par une lettre de dénonciation sous cet angle: n'y a-t-il pas allusion à des pratiques attribuées, dans le discours dont on a retrouvé la trace, aux régimes communistes?

Ainsi est-ce un va-et-vient entre le contexte (rapprochement opéré entre des termes appartenant à la même classe-objet) et la mémoire discursive (trace d'un discours anticommuniste qui permet de reconsidérer la valeur de l'ensemble des termes), qui détermine la valeur négative du syntagme centralisme démocratique et de la classe-objet. De plus est créée par là une certaine image des interlocuteurs-adversaires - et plus loin ils seront traités d'apparatchiks. On peut alors se demander si le véritable objet du conflit qui oppose les deux parties ne serait pas finalement d'ordre politique.

\section{Conclusion}

Nous sommes partie d'un aspect important de notre recherche sur la construction de l'objet de discours, la question du désignateur. Ce type de question n'est bien entendu pas inconnu des études argumentatives ${ }^{45}$. Cependant, conformément au cadre psychologique qui est en général le sien, ce genre de travaux a tendance à mettre en avant le caractère intentionnel d'un choix qui correspondrait alors à une "stratégie ${ }^{46}$. Or, refusant les 
spéculations sur une quelconque intentionalité, nous avons voulu partir des effets discursifs liés à la présence de tel ou tel désignateur. Un premier effet peut apparaître, le plus évident: la valeur d'appréciation négative du désignateur. Les termes employés ( questions de dernière minute, centralisme démocratique/diktat/.., dégraissage) justifient alors dans une certaine mesure la prise de position du locuteur vis-à-vis de l'objet. Avec ce type d'analyse on reste dans un cadre argumentatif, et on ne prend pas en compte l'ensemble des désignateurs (décision du conseil de classe ne comporte pas de visée appréciative).

41 Prenant alors en considération la forme syntaxique de certains des désignateurs rencontrés - relative, nominalisation - on a fait le rapprochement avec un type de recherches propres à l'AD française, s'intéressant aux traces, dans l'énoncé, de l'interdiscours et de la mémoire discursive. On a cherché à caractériser un deuxième type d'effet: dans la constitution de l'objet de discours, par la présence de tel ou tel désignateur, ce n'est pas «seulement » une valeur positive ou négative qui est imposée, mais tout le poids d'un autre discours ${ }^{47}$. Cependant, l'articulation de notions issues d'ensembles théoriques différents n'est pas sans soulever un certain nombre de questions.

42 Une question importante a trait au mode de constitution du corpus. Si l'on peut parler de filiation entre les notions d'« interdiscours » et de "mémoire discursive », il n'y a pas cependant superposition entre les deux. Alors que l'interdiscours est défini de façon relativement abstraite ${ }^{48}$ et qu'il ne semble pas envisageable de se donner les moyens de l'appréhender concrètement ${ }^{49}$, la notion de "domaine de mémoire", elle, repose précisément sur la possibilité de rassembler des éléments (discours, énoncés) qui permettent de saisir la « mémoire discursive » d'un discours, ou d'un énoncé. Un pont est alors tendu vers Bakhtine et la notion de « dialogisme », ainsi que le souligne J. Authier ${ }^{50}$, qui propose, elle, le concept plus général de «non-coïncidence » discursive.

43 Mais le corpus que nous utilisons ici, parce qu'il est construit avec un objectif différent, ne permet pas cette «saisie » de l'interdiscours. On peut d'ailleurs poser la question du matériel qu'il serait nécessaire de recueillir dans ce but. Dans le cas du lycée, sans doute faudrait-il avoir accès aux textes administratifs, mais on devrait également prendre en compte leur mode de diffusion auprès des proviseurs (stages, réunions, ...) et s'intéresser aussi sans doute aux discussions en salle des professeurs ... Dans certains cas (la valeur de centralisme démocratique par exemple), il serait indispensable de travailler également sur le discours médiatique, dans la mesure où il nous semble contribuer à forger une sorte $\mathrm{d}^{\prime}$ « opinion moyenne » sur un certain nombre d'objets (ici le communisme, ou les régimes communistes).

La deuxième question que nous soulèverons concerne plus directement une recherche axée sur le mode de constitution de l'objet de discours. La prise en compte des préconstruits sous-jacents à l'objet de discours a en effet mis en évidence un «second fond ", un "arrière-plan » de cet objet. Ainsi le proviseur refusant de répondre aux questions de dernière minute se réfère-t-il à un mode de fonctionnement de l'instance « conseil d'administration » qu'il préside. De même, le membre de l'association dont nous avons cité les paroles reproche à ses adversaires d'agir sur le mode du centralisme démocratique, tandis que le délégué syndical, parlant de dégraissage, porte la discussion sur le terrain - balisé - de l'opposition « travailleurs »-« patronat ». Partant de là, ne peut-on faire l'hypothèse qu'à côté de l'objet explicitement posé, il y en aurait un autre, beaucoup plus lié au cadre institutionnel dans lequel prend place la réunion? Reste la question des marques permettant de repérer cet « objet caché »... 


\section{BIBLIOGRAPHIE}

AUTHIER-REVUZ, J. (1992) Les non-coïncidences du dire et leur représentation méta-énonciative. Etude linguistique et discursive de la modalisation autonomique. Thèse de Doctorat d'Etat, soutenue le 16 mai 1992 à l'Université de Paris VIII.

AUTHIER-REVUZ, J. (1988) Non-coïncidences énonciatives dans la production du sens, LINX, 19, p. 25-28.

AUTHIER-REVUZ, J. (1982) Hétérogénéité montrée et hétérogénéité constitutive, éléments pour une approche de l'autre dans le discours, DRLAV, 26, p. 91-151.

BOREL, M.J., GRIZE, J.B., MIÉVILLE, D. (1992) Essai de logique naturelle, Berne, Peter Lang, (2ème éd. : 1ère éd. 1983).

COURTINE, J.J. (1981) Quelques problèmes théoriques et méthodologiques en analyse du discours, à propos du discours communiste adressé aux chrétiens, Langages, 62, juin 1981.

FUCHS, C. (1987) Les relatives et la construction de l'interprétation, Langages, 88, p.95-127.

GRIZE, J.B. (1990) Logique et langage, Paris, Ophrys.

GRIZE, J.B. éd. (1984) Sémiologie du raisonnement, Berne, Peter Lang.

GUILHAUMOU, J. et MALDIDIER, D. (1979) Courte critique pour une longue histoire. L'analyse du discours ou les (mal)heures de l'analogie, Dialectiques, 26, p. 7-23.

LECOMTE, A. (1981) Comment Einstein raconte comment Newton expliquait la lumière (ou : le rôle de la mémoire interdiscursive dans le processus explicatif), Revue Européenne des Sciences Sociales, tome XIX, n56, p. 69-93.

LECOMTE, A. (1983) Raisonner : quand dire c'est faire voir, Travaux de centre de recherches sémiologiques de Neuchâtel, $\mathrm{n}^{\circ} 44$.

MALDIDIER, D. (1993) L'inquiétude du discours, un trajet dans l'histoire de l'analyse du discours : le travail de Michel Pêcheux, Semen 8, p. 107-119.

MALDIDIER, D. et PÊCHEUX, M. (1990) L'inquiétude du discours, Editions des cendres, Paris.

MONDADA, L. (1994), Verbalisation de l'espace et fabrication du savoir Approche linguistique de la construction des objets de discours, Lausanne.

MOREL, M.A. (1992) Intonation et thématisation, L'information grammaticale, 54, p. 26-35.

MOREL, M.A. et RIALLAND, A. (1993) L'énoncé oral complexe. Les relatives en qui, Travaux de linguistique du CERLICO nº, Subordination-Subordinations II, Presses Universitaires de Rennes II.

MORTUREUX, M.F. (1994) Comment peut-on définir la propriété d'un teme, in Moirand et alii, Parcours linguistiques de discours spécialisés, Actes du colloque, 23-25 septembre 1992, Sorbonne, Berne, Peter Lang, p. 3-10.

PÊCHEUX, Analyse du discours : trois époques, in Maldidier/Pêcheux, 1990, p. 295-302.

PLANTIN, C. (1991) Question -> Argumentations -> Réponses, in La Question (sous la direction de C. Kerbrat-Orecchioni), Lyon, PUL, p. 63-85.

PLANTIN, C. (1993) Situation rhétorique, Verbum, 1-2-3, p. 229-239. 
SÉRIOT, P. (1986) Langue russe et discours politique soviétique : analyse des nominalisations,

Langages 81, p.11-41.

Langages, 117, mars 1995, Les analyses de discours en France.

Les Carnets du Cediscor, 3, (1995) Les enjeux des discours spécialisés, sous la direction de J.C Beacco et S. Moirand, Presses de la Sorbonne Nouvelle.

\section{ANNEXES}

\section{Annexe 1 : conventions de transcription}

- - marque le début d'un énoncé

/ note une pause

(h) note une pause remplie par une inspiration audible

e note une hésitation (euh dans la graphie traditionnelle)

: note l'allongement d'un son

MAJ les caractères en majuscules indiquent une prononciation appuyées

= signale qu'un mot est inachevé

<.> indique qu'un segment n'a pu être transcris

(MAJ) décrit un mode d'articulation. Précède l'énoncé qu'il caractérise et reste valable jusqu'au signe +

P, G les différents locuteurs

\section{Annexe 2 : intervention initiale de $P\left(\operatorname{cas~} n^{\circ} 1\right)$}

P - - (...) nous av=j'ai / reçu // quatre questions de la fcpe et une question // sur le cdi /// alors / puisque je on commence e à travailler avec le nouveau conseil d'administration / je voudrais quand même e donner un certain nombre de : de remarques et de dispositions /// je ne crois pas personnellement / à la valeur des questions de dernière minute qui ont pas pu être étudiées préalablement / et c'est tellement vrai que la réglementation en vigueur / m'empêche de mettre au e à l'ordre du jour du conseil d'adminsitration des questions d'ordre pédagogique qui n'auraient pas été étudiées préalablement en commission permanente /// c'est l'article deux du décret du trente août modifié /// alors c'est bien aussi / qu'on puisse e s'exprimer pleinement au conseil d'administration mais je crois qu'un conseil d'administration doit être préparé / moi je CROIS à la valeur des commissions permanentes c'est comme si vous voulez si on allait TRAvailler sur le règlement intérieur ici comment voulez-vous qu'on travaille à trente plus de trente donc il faut un énorme travail préalable et ensuite on propose un texte et on travaille sur ce texte on l'amende on e on s'y oppose on change on modifie on refuse on accepte mais un peu comme au parlement si vous voulez il faut un travail 
préalable / alors je sais bien que le mercredi à la chambre des députés il y a bien le les questions diverses / mais e pf vous connaissez tout l'intérêt des questions diverses à la chambre des députés et je voudrais pas qu'on se transforme en $\mathrm{ch}=$ en mercredi de la chambre des députés / alors e : : entres autres la fcpe donc me propose les heures d'option musique et natation pour le bac ont-elles été récupérées et seront-elles reconduites pour les années à venir // moi je répondrai pas à cette question-là parce que donc je peux pas dans le sens que e il y a pas d'étude préalable qui a été faite et ensuite il faut / voir / ça en commission permanente

\section{Annexe 3 : contextes d'apparition des éléments de la classe-objet ouverte par la lettre (cas $n^{\circ} 3$ )}

1. B ... suite à une première lettre / on a essayé de ne pas jouer cette mécanique e qui était annoncée dans la lettre

2. B ... que la lettre ait eu cet effet e aussi e aussi fort

3. A ... j'ai absolument pas compris la signification de cette lettre de septembre

4. A ... et que ce procédé e le procédé utilisé nous a paru complètement e e archaïque anachronique e à la limite de la : (h) e : à la limite de la : (ff) je dirais de la raison

5. A ... d'envoyer une lettre de dénonciation

6. A ... si e cette lettre a dépassé ... votre pensée

7. A ... cette lettre a été écrite

8. A ... la manière de fonctionner dans un mouvement comme le nôtre / est le combat le débat / le débat la : la discussion l'échange le : (h) les interrogations / MAIS certainement pas le centralisme démocratique / qui : est en train de s'écrouler partout

9. A ... vous avez envoyé la lettre la lettre

10. A ... qu'est-ce que ça veut dire que : : qu'un : qu'un diktat disant

11. A ... cette accusation grave que vous nous faites / sans preuves

12. A ... on s'est pas permis d'écrire aucune lettre

13. A ... et qu'est-ce que vous espériez par cette lettre

14. B ... il y a eu e notre rupture en septembre

15. B ... s'il y avait pas eu la rupture de septembre

repris par A ... mais qui a fait cette rupture en septembre

16. B ... pourquoi cette lettre de septembre

17. B ... c'est (h) pour expliquer un petit peu la l= la lettre de septembre

18. A ... l'argument d'autorité qui consiste à dire

19. A ... bah si c'est c'est votre lettre

20. A ... tu la relis cette lettre / mais qu'est-ce que c'est que ça si c'est qu'argument d'autorité et juridique / de type stalinien je dirais même

21. B ...cette lettre ne ressorte pas à chaque argument 
22. B ... le malaise date pas de cette lettre-là

23. B ... il faut arrêter de sortir cette lettre aujourd'hui

\section{NOTES}

1. Comme en témoigne le titre du dernier numéro de Langages (117, mars 1995) consacré au sujet (Les analyses de discours en France), et l'introduction de D. Maingueneau dans ce même numéro.

2. Présentation remise en question par $C$. Normand lors de la journée organisée par le CEDISCOR à l'occasion de la parution du numéro 117 de Langages (23 juin 1995, Université de la Sorbonne nouvelle).

3. 3 Même si les derniers textes de M. Pêcheux évoquent la possibilité de travailler sur du « conversationnel ». Voir « Lecture et mémoire : projet de recherche », in Maldidier, 1990, 290, note 2: «L'analyse de discours de type français a essentiellement thématisé les discursivités textuelles, en laissant de côté les discursivités orales. Mais il n'y a aucune raison théorique de ne pas se poser aussi la question de l'interdiscours conversationnel ». On se reportera également à «Analyse du discours : trois époques », ibid, 301.

4. On peut attribuer cette situation en partie à la diffusion des travaux faits en ethnométhodologie, mais peut-être aussi aux recherches portant sur la description de l'oral (citons le GARS à Aix, le Centre de Recherches sur le français contemporain à Paris III, les travaux de F. Gadet à Paris-X).

5. Cette notion est définie par Plantin, 1991 et 1993 : autour d'une " question argumentative » (qui structure une "situation argumentative»), s'affrontent proposition et contre-proposition, argumentaire et contre-argumentaire. La situation argumentative se caractérise également par le fait qu'elle met en jeu trois protagonistes, non seulement le tenant de chacune des positions, mais encore un tiers, médiateur ou juge.

6. Voir en bibliographie les références des travaux menés autour de J.B. Grize au Centre de Recherches sémiologiques de l'Université de Neuchâtel. Il conviendrait d'expliciter cette référence à la logique naturelle. Signalons simplement ici que notre conception de l'objet de discours est « discursive » et non pas « cognitive ».

7. Grize éd, 1992, 4.

8. Grize éd, 1992, 78.

9. La classe-objet est plus riche qu'un classe distributive car les éléments qui la composent peuvent être hétérogènes : elle "possède certaines des propriétés de la classe méréologique » (Miéville in Grize éd, 1984, 236).

10. Cette question est récurrente. Déjà posée par Courtine, 1981 : «Comment, dans le discours luimême et par le discours lui-même, un élément déterminé peut-il être caractérisé comme thème de discours » (79), elle est reprise, par exemple, par Mondada, 1992 et 1994, qui cherche à établir une liste des marques d'introduction du « topic » dans un corpus oral « conversationnel » et dans un corpus écrit de relations de voyage.

11. Ce type de marques est en relation avec la constitution du corpus autour d'une "question argumentative » : c'est la position du locuteur par rapport à l'objet, repérée en particulier par la relation prédicative.

12. L'objet est repris par les interlocuteurs et donne lieu à de nouvelles prédications.

13. Nous suivons ici pour l'essentiel la présentation proposée par D. Maldidier en introduction au recueil de textes de Pêcheux (Maldidier, 1990).

14. Mené en parallèle avec P. Henry. Cf. P. Henry, "Constructions relatives et articulations discursives », Langages, 37, 1975. La notion de préconstruit est complétée par celle d'articulation d'énoncés. 
15. Courtine, 1981.

16. La notion de "mémoire discursive " paraît «sous-jacente à l'analyse des FD [formations discursives] qu'effectue l'Archéologie» (Courtine, 1981, 52) et la notion de «domaine de mémoire» est un terme que l'on trouve dans L'archéologie, avec "domaine d'actualité », «domaine d'anticipation", mais « avec une valeur sensiblement différente » (ibid., 55, note 2). Voir également Authier, 1994, t. 1, 127.

17. «Un bougé vient de l'hésitation qui semble affecter l'interdiscours, dès lors que le concept de "domaine de mémoire" introduit par J. J. Courtine, à la suite de M. Foucault, entre en concurrence avec lui et en paraît parfois le simple substitut. Remaniement important, eu égard aux positions de départ sur la question du sujet, mais qui permet peut-être, en affaiblissant le concept, de le faire travailler et d'esquisser un déblocage du côté du sujet » (Maldidier, 1993, 118).

18. Cet extrait ainsi que le suivant est tiré du conseil d'administration d'un lycée, en grande partie consacré à l'adoption d'un nouveau règlement intérieur. Ici, le proviseur vient de donner la liste des différents points de l'ordre du jour.

19. On trouvera en annexe l'intégralité de l'intervention ainsi que la liste des conventions de transcription. Nous ne revenons pas sur l'inépuisable question de la transcription, nous contentant de renvoyer à la contribution de $\mathrm{S}$. Wachs ici-même.

20. Ce «travail» de l'objet de discours aboutit à la recatégorisation de question en communication, acceptable par P car elle supprime la valeur de contrainte illocutoire liée au premier terme.

21. Ou encore l'« étiquette » de la classe : cf Borel, 1984, 181, pour qui « un des indicateurs (est-ce nécessairement le premier ?) de la liste d'une classe-objet peut, quand il le fait, indiquer un "générateur" (il peut y en avoir plus d'un) ou l' "étiquette" de la classe ".

22. On opposera ainsi quatre, une à les; j'ai reçu à je ne crois pas; de la fcpe, sur le cdi à de dernière minute.

23. Il s'agirait alors du «mot juste» (Mortureux, 1993) ou encore du " dénominateur» (Kleiber, 1984), même si le syntagme questions diverses n'est pas répertorié par le dictionnaire consulté (Le Petit Robert).

24. En ce sens le «mot juste » est aussi le terme «neutre » relativement à l'appréciation. Sur la notion d'appréciation, voir Les Carnets du CEDISCOR, 3.

25. Il n'y a rien en soi de négatif dans "un invité de dernière minute », ou " une nouvelle de dernière minute ".

26. Nous procédons à une écoute à l'oreille, la qualité de l'enregistrement ne permettant pas une analyse au moyen des instruments adéquats.

27. Morel et Rialland, 1993, 17. La forme de l'antécédent irait également dans ce sens (note 7, ibid ). Fuchs, 1987 montre bien le caractère réducteur de l'opposition «descriptive" (ou appositive)/ « restrictive» (ou «déterminative»). Dans le cas présent cependant, outre les indices de type intonatif, une lecture du type : "parmi les questions de dernière minute, il y a celles qui n'ont pas pu être étudiées et les autres" nous semble peu probable. Pour nous, l'interprétation « appositive » peut donc ici se justifier.

28. Pêcheux, 1975, in Maldidier, 1990, 231. Sur le caractère d'évidence lié au fonctionnement appositif de la relative, voir également Henry, 1975, 98.

29. On constate par ailleurs que, dans la suite, les interventions de $P$ thématisent un objet de discours en relation précisément avec le mode de fonctionnement de l'instance.

30. On sait que la nominalisation a également pour effet d'effacer l'agent, l'aspect verbal et la modalité de l'énoncé antérieur.

31. Il s'agit de la même réunion que l'extrait 1 . Les participants examinent les différents articles du règlement intérieur. C'est un professeur qui prend la parole. 
32. On constate d'ailleurs que dans la suite de la séquence est thématisé par ce professeur non pas la décision du conseil de classe ou le conseil de classe décide - objet « impossible » - mais plutôt un énoncé du type le conseil de classe a le droit de s'exprimer.

33. Il s'agit d'un extrait du comité d'entreprise au cours duquel les participants - délégués syndicaux et direction - discutent le point du l'ordre du jour intitulé " point sur les congés de fin de carrière ». Le délégué CGT dont nous reproduisons les paroles met en doute le caractère volontaire des départs. Signalons que les difficultés économiques de l'entreprise laissent craindre des licenciements, bien qu'aucun plan n'ait été annoncé par la direction qui dément toute rumeur.

34. On pourrait également considérer, au vu des thématisations, que l'objet est représenté par les personnes que l'on contacte et à qui l'on demande ....

35. Préparé par : donc on dégraisse tranquillement comme ça et repris par si si c'est un dégraissage.

36. On peut aussi parler de « classification » : cf Borel, 1992, 184.

37. «Certains discours contiennent des marques, telles que les démonstratifs (...), qui compactifient cet espace» (Lecomte, 1983, 15). Des phénomènes analogues ont également été décrits par Mortureux. Du côté de la syntaxe de l'oral, M.A. Morel souligne la valeur conclusive liée à la forme en c'est - valeur renforcée ici par la présence du ligateur en fait (Morel, "Distribution des présentatifs dans des dialogues finalisés ", Première publication du Colloque sur la Deixis, janvier 1990, publié in Structuration linguistique du dialogue oral, Document de travail $n^{\circ} 1$, Publications de la Sorbonne Nouvelle, 1991/1992, 104).

38. Le Petit Robert donne pour dégraissage : « action de dégraisser. Son résultat. Le dégraissage d'un vêtement. V. nettoyage ». Par contre, pour dégraisser, on trouve à la fin de l'article : "Alléger les frais, effectuer des économies (notamment en licenciant le personnel)».

39. Répondant au « on » de on dégraisse.

40. Il s'agit d'une réunion de conciliation entre deux parties, A et B, qui sont membres d'une association d'éducation populaire (l'une représente le département et l'autre la commune). A la suite d'une lettre particulièrement agressive envoyée par la première à la seconde, les relations ont été suspendues entre les deux parties. C'est précisément de cette lettre qu'il s'agit ici.

41. En voici la liste: \{une première lettre, la lettre, notre rupture, la rupture de septembre, cette lettre de septembre, cette lettre, cette lettre-là\}. La seule redésignation substitue rupture à lettre, mais la rupture est ici la conséquence objectivement constatée de l'envoi de la lettre.

42. Classe-objet plutôt que paradigme désignationnel : ce dernier terme restreint aux termes en relation de co-référence, tandis que la classe-objet accueille les objets contigus.

43. On trouvera en annexe les contextes d'emploi de chaque terme.

44. Voici ce que dit Le Petit Robert: «stalinien: de Staline, propre à Staline, au stalinisme. La dictature stalinienne. Méthodes staliniennes. Partisans de Staline et du stalinisme (spéclt. qui reste fidèle à l'esprit stalinien malgré la déstalinisation)»; «stalinisme: politique stalinienne d'autorité, de contrainte. Théories et méthodes de Staline ».

45. Plantin montre comment, dans une discussion sur l'avortement, «les partisans de l'avortement désignaient le ${ }^{* * *}$ par le mot foetus alors que les adversaires de l'avortement désignaient ce même *** par le mot bébé » (Essais sur l'argumentation, Paris, Kimé, 1990, 233).

46. Ainsi Plantin parle-t-il $\mathrm{d}^{\prime}$ '«objets irréductiblement marqués par des positions intentionnelles » (ibid).

47. Grize s'intéresse également, bien évidemment, au choix du terme initial dans ce qu'il appelle «l'opération d'ancrage», qui ancre un objet dans une "notion primitive ». Commentant un exemple, il indique : "quant à la notion primitive $\mathrm{X}$, nous avons déjà remarqué qu'il était impossible d'en "dire" quoi que ce soit. En revanche, que Mességué ait choisi le nom "médecin", plutôt que "toubib" ou "praticien", est significatif. Par le fait même qu'il s'agit de mots en langue naturelle, ils évoquent nécessairement tout un ensemble de propriétés et de relations, ils sont engagés dans un faisceau de lieux communs » (in Borel et alii, 1992, 105-106). La 
perspective de la logique naturelle est cognitive tandis que la nôtre est discursive. C'est en cela quenous ne nous inscrivons pas totalement dans le cadre théorique élaboré par J.B. Grize (voir note 6).

48. «L'interdiscours n'est ni la désignation banale des discours qui ont existé avant ni l'idée de quelque chose de commun à tous les discours. Dans un langage strictement althussérien, il est le 'tout complexe à dominante' des formations discursives, intriqué dans le complexe des formations idéologiques » (Maldidier, 1990, 43).

49. "Quand nous disons que la production d'un sens d'une séquence discursive repose sur la possibilité de rapporter cette séquence à une formation discursive, il est bien évident que nous ne voulons pas dire que dans la lecture d'un texte, il soit nécessaire de le confronter matériellement avec un autre texte" (Henry, op. cit., 96). Voir également Authier, 1994, t. 1, 126.

50. "Cette démarche donne corps, de façon concrète, à l'approche bakhtinienne des mots du discours "chargés", "occupés", "saturés", ... des mots des autres" (Authier, op. cit., 128).

\section{RÉSUMÉS}

On pose la question de l'articulation de la notion d'objet de discours, telle qu'elle est conçue en logique naturelle, avec des notions issues de l'Analyse de Discours française. On montre, sur des exemples tirés d'un corpus de situations argumentatives orales, comment on peut faire intervenir, dans l'analyse de la désignation de l'objet de discours, les notions de préconstruit, d'interdiscours, de domaine de mémoire.

This article examines the question of the articulation of the idea of « object of discourse » as it is perceived in «natural logic » (J. B. Grize) according to the principles set forth by the French Discourse Analysis. It uses examples from a corpus of argumentative oral situations to demonstrate how the notions of « preconstruction ", « interdiscourse » (Pêcheux) and « domain of memeory » (Foucault) may be used in the analysis of the designation of the object of discourse.

\section{AUTEUR}

\section{FRÉDÉRIQUE SITRI}

